\title{
Two new aliphatic lactones from the fruits of Coriandrum sativum L.
}

\author{
Kamran J Naquvi, Mohammed Ali and Javed Ahmad
}

\begin{abstract}
Background: The present paper describes the isolation and characterization of two new aliphatic $\delta$-lactones along with three glycerides and n-nonadecanyl cetoleate from the fruits of Coriandrum sativum L. (Apiaceae). The structures of all the isolated phytoconstituents have been established on the basis of spectral data analysis and chemical reactions.

Results: Phytochemical investigation of the methanolic extract of $C$. sativum L. (Apiaceae) fruits resulted in the isolation of two new aliphatic $\delta$-lactones characterized as $2 a-n$-heptatriacont-(Z)-3-en-1,5-olide (1) (coriander lactone) and 2a-n-tetracont-(Z,Z)-3,26-dien-18a-ol-1,5-olide (2) (hydroxy coriander lactone) together with glyceryl1,2-dioctadec-9,12-dienoate-3-octadec-9-enoate (3); glyceryl-1,2,3-trioctadecanoate (4); n-nonadecanyl-n-docos-11enoate (5) and oleiyl glucoside (6).
\end{abstract}

Conclusions: Phytochemical investigation of the methanolic extract of $C$. sativum gave coriander lactone and hydroxy coriander lactone as the new phytoconstituents.

Keywords: Coriandrum sativum, Apiaceae, Aliphatic $\delta$-lactones, Fatty acid glycerides

\section{Background}

Coriandrum sativum $\mathrm{L}$. is an annual and herbaceous plant belonging to the Apiaceae family. It is a medicinal plant, native of southern Europe and western Mediterranean region and is cultivated worldwide [1]. India is the largest producer of coriander in the world. Major production centers are Rajasthan, Maharastra, Gujarat, and Karnataka [2]. The whole plant and especially the unripe fruits are characterized by a strong disagreeable odor, hence the name coriander, giving characteristic aroma when rubbed [3]. The most important constituents of coriander seeds are the essential oil and the fatty oil. The dried coriander seeds contain an essential oil $(0.03 \%$ to $2.6 \%$ ) with linalool as main component $[4,5]$, phenolics, flavonoides [6], and isocoumarin compounds [7]. It has traditionally been referred to as antidiabetic [8] and cholesterol lowering drug $[9,10]$. This paper describes the isolation and characterization of two new aliphatic $\delta$-lactones along with fatty acid glycerides, ester, and glucoside from the fruits of $C$. sativum of Delhi region of India.

\footnotetext{
* Correspondence: maliphyto@gmail.com
Department of Pharmacognosy and Phytoch

* Correspondence: maliphyto@gmail.com
Department of Pharmacognosy and Phytochemistry, Faculty of Pharmacy, Jamia Hamdard, New Delhi 110062, India
}

\section{Methods}

General

Melting points were determined on a Perfit melting apparatus (Ambala, Haryana, and India) and are uncorrected. UV spectra were measured with a Lambda Bio 20 spectrophotometer (Perkin-Elmer-Rotkreuz, Switzerland) in methanol. Infrared spectra were recorded on Bio-Rad FTIR 5000 (FTS 135, Kowloon, Hong Kong) spectrophotometer using $\mathrm{KBr}$ pellets; $\gamma_{\max }$ values are given in $\mathrm{cm}^{-1}$. ${ }^{1} \mathrm{H}$ nuclear magnetic resonance (NMR) and ${ }^{13} \mathrm{C}$ NMR spectra were screened on Bruker spectrospin 300 and $75 \mathrm{MHz}$, respectively, instruments (Karlesruthe, Germany) using $\mathrm{CDCl}_{3}$ and TMS as an internal standard. Mass spectra were measured by effecting fast atom bombardment (FAB) ionization at $70 \mathrm{eV}$ on a JEOL-JMS-DX 303 spectrometer (JEOL, Japan) equipped with direct inlet probe system. Column chromatography was performed on silica gel (60 to 120 mesh; Qualigen, Mumbai, India). TLC was run on silica gel G (Qualigen, Carlsbad, CA). Spots were visualized by exposing to iodine vapors, UV radiation, and spraying with ceric sulphate.

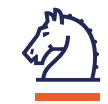

(C) 2012 Naquvi et al.; licensee Springer. This is an Open Access article distributed under the terms of the Creative Commons Attribution License (http://creativecommons.org/licenses/by/2.0), which permits unrestricted use, distribution, and reproduction in any medium, provided the original work is properly cited. 


\section{Results and discussion}

Compound 1, named coriander lactone (Scheme 1), was obtained as a pale yellow crystalline mass from petroleum ether eluents. Its IR spectrum showed characteristic absorption bands for $\delta$-lactone group $\left(1738 \mathrm{~cm}^{-1}\right)$, unsaturation $\left(1621 \mathrm{~cm}^{-1}\right)$, and long aliphatic chain (806, $721 \mathrm{~cm}^{-1}$ ). On the basis of FAB mass spectrum, its molecular weight was established at $\mathrm{m} / z 546$ consistent with the molecular formula of an unsaturated lactone, $\mathrm{C}_{37} \mathrm{H}_{70} \mathrm{O}_{2}$. It indicated three double bond equivalents; two of them were adjusted in the lactone ring and remaining one in the vinylic linkage. The prominent ion peaks arising at $\mathrm{m} / z$ $97\left[\mathrm{C}_{2}-\mathrm{C}_{6} \text { fission, } \mathrm{C}_{5} \mathrm{H}_{5} \mathrm{O}_{2}\right]^{+}$and 449 [M-97, $\left.\left(\mathrm{CH}_{2}\right)_{31} \mathrm{CH}_{3}\right]^{+}$suggested that $\delta$ lactone was attached to the $\mathrm{C}-32$ aliphatic chain. The ${ }^{1} \mathrm{H}$ NMR spectrum of 1 showed a one-proton double doublet at $\delta$ $5.36(J=6.6,7.2 \mathrm{~Hz})$ and a one-proton multiplet at $\delta$ 5.32 assigned to $(\mathrm{Z})$-vinylic $\mathrm{H}-3$ and $\mathrm{H}-4$ protons, respectively. A two-proton doublet at $\delta 4.14(J=7.2 \mathrm{~Hz})$ was ascribed to oxygenated methylene $\mathrm{H}_{2}-5$ protons. A one-proton broad multiplet at $\delta 2.78$ with half width of $6.1 \mathrm{~Hz}$ was attributed to $\beta$-oriented $\mathrm{H}-2$ methine proton. A two-proton multiplet at $\delta 2.30$, two four-proton multiplets at $\delta 2.05$ and 1.59 , and a broad signal at $\delta 1.26$ integrating for 52 protons were associated with the methylene protons. A three-proton triplet at $\delta 0.90$ $(J=6.3)$ was accounted to C-37 primary methyl protons. The ${ }^{13} \mathrm{C}$ NMR spectrum of $\mathbf{1}$ displayed signals for lactone carbon at $\delta 173.56(\mathrm{C}-1)$, vinylic carbons at $\delta$ 130.15 (C-3) and 127.91 (C-4), oxygenated methylene carbon $\delta 66.83(\mathrm{C}-5)$, methine carbon at $\delta 34.21(\mathrm{C}-2)$, methylene carbons between $\delta 31.91$ and 22.64, and methyl carbon at $\delta 14.03(\mathrm{C}-37)$. The ${ }^{1} \mathrm{H}^{-1}{ }^{1} \mathrm{H}$ correlation spectroscopy (COSY) spectrum of $\mathbf{1}$ showed correlations of $\mathrm{H}_{2}-5$ with $\mathrm{H}-4$ and $\mathrm{H}-3$; and $\mathrm{H}-2$ with $\mathrm{H}-3, \mathrm{H}-4$ and $\mathrm{H}_{2}-6$. The heteronuclear multiple bond correlation (HMBC) spectrum of $\mathbf{1}$ exhibited interactions of $\mathrm{C}-1$ with $\mathrm{H}-2, \mathrm{H}-3$ and $\mathrm{H}_{2}-6$; and $\mathrm{C}-5$ with $\mathrm{H}-4$ and $\mathrm{H}-3$. On the basis of the foregoing account, the structure of $\mathbf{1}$ has been established as 2- $\alpha$-n-heptatriacont-(Z)-3-en-1,5olide. This is a new $\delta$-lactone isolated from a plant source [see Additional file 1].

Compound 2, named hydroxy coriander lactone, was obtained as a pale yellow crystalline mass from chloroform eluents (Scheme 2). Its IR spectrum showed distinctive absorption bands for a hydroxy group $\left(3463 \mathrm{~cm}^{-1}\right)$, lactone ring $\left(1743 \mathrm{~cm}^{-1}\right)$, unsaturation $\left(1645 \mathrm{~cm}^{-1}\right)$, and long aliphatic chain $\left(722 \mathrm{~cm}^{-1}\right)$. Its FAB mass spectrum had a molecular ion peak at $m / z 603[\mathrm{M}+\mathrm{H}]^{+}$corresponding to a long chain hydroxylated aliphatic lactone, $\mathrm{C}_{40} \mathrm{H}_{75} \mathrm{O}_{3}$. It showed four degrees of unsaturation; two each of them were adjusted in the lactone ring and two in vinylic linkages. The prominent ion peaks arising at $\mathrm{m} / z 97\left[\mathrm{C}_{2}-\mathrm{C}_{6}\right.$ fission, $\left.\mathrm{C}_{5} \mathrm{H}_{5} \mathrm{O}_{2}\right]^{+}, 265\left[\mathrm{C}_{17}-\mathrm{C}_{18} \text { fission, } \mathrm{C}_{5} \mathrm{H}_{5} \mathrm{O}_{2}\left(\mathrm{CH}_{2}\right)_{12}\right]^{+}$,
$337\left[\mathrm{M}-265, \quad \mathrm{HOCH}\left(\mathrm{CH}_{2}\right)_{7} \mathrm{CH}=\mathrm{CH}\left(\mathrm{CH}_{2}\right)_{12} \mathrm{CH}_{3}\right]^{+}, \quad 295$ $\left[\mathrm{C}_{18}-\mathrm{C}_{19} \text { fission, } \mathrm{C}_{5} \mathrm{H}_{5} \mathrm{O}_{2}\left(\mathrm{CH}_{2}\right)_{12} \mathrm{CHOH}\right]^{+}$suggested the presence of one of the vinylic linkage in the lactone ring and the hydroxyl group at $\mathrm{C}-18$. The ion peak forming at $m / z 393\left[\mathrm{C}_{25}-\mathrm{C}_{26} \text { fission, } \mathrm{C}_{5} \mathrm{H}_{5} \mathrm{O}_{2}\left(\mathrm{CH}_{2}\right)_{12} \mathrm{CHOH}\left(\mathrm{CH}_{2}\right)_{7}\right]^{+}$ indicated the location of another vinylic linkage at $\mathrm{C}-26$. The ${ }^{1} \mathrm{H}$ NMR spectrum of $\mathbf{2}$ showed a one-proton double doublet at $\delta 5.34(6.8,7.5 \mathrm{~Hz})$ and four multiplets at 5.26, $5.17\left(\mathrm{w}_{1 / 2}=8.5 \mathrm{~Hz}\right)$, and $5.11\left(\mathrm{w}_{1 / 2}=9.5 \mathrm{~Hz}\right)$ assigned correspondingly to cis-oriented $\mathrm{H}-4, \mathrm{H}-3, \mathrm{H}-26$, and $\mathrm{H}-27$ vinylic protons, respectively. Two one-proton doublets at $\delta$ $4.32(J=7.5 \mathrm{~Hz})$ and $4.28(J=7.5 \mathrm{~Hz})$ were attributed to the oxygenated methylene $\mathrm{H}_{2}-5$. A one-proton broad multiplets at $\delta 4.16$ with half width of $5.7 \mathrm{~Hz}$ was ascribed to $\beta$ oriented $\mathrm{H}-18$ carbinol proton. A one-proton multiplet at $\delta$ $2.76\left(\mathrm{w}_{1 / 2}=6.8 \mathrm{~Hz}\right)$ was accounted to $\beta$-oriented $\mathrm{H}-2$ methine protons. Two multiplets at $\delta 2.30$ and 2.02 integrating for two protons each were due to methylene $\mathrm{H}_{2}-25$ and $\mathrm{H}_{2}-28$ protons located nearby the $\mathrm{C}-26$ and $\mathrm{C}-27$ vinylic carbons. The remaining methylene protons resonated between $\delta 1.98$ and 1.26. A three-proton triplet at $\delta$ $0.87(J=6.5 \mathrm{~Hz})$ was associated with the C-40 primary methyl protons. The ${ }^{13} \mathrm{C}$ NMR spectrum of $\mathbf{2}$ displayed signals for lactone carbon at $\delta 172.78(\mathrm{C}-1)$, vinylic carbons at $\delta$ 130.34 (C-4), 129.78 (C-3), 128.78 (C-26), and 127.96 (C$27)$, carbinol carbon at $\delta 68.83$ (C-18), oxygenated methylene carbon at $\delta 65.95$ (C-5), methine carbon at $\delta 42.03$ (C2), methylene carbons between $\delta 33.76$ and 22.55 , and methyl carbon at $\delta 13.94(\mathrm{C}-40)$. The ${ }^{1} \mathrm{H}_{-}{ }^{1} \mathrm{H}$ COSY spectrum of 2 showed correlations of $\mathrm{H}_{2}-5$ with $\mathrm{H}-4$ and $\mathrm{H}-3 ; \mathrm{H}-2$ with $\mathrm{H}-3, \mathrm{H}-4$, and $\mathrm{H}_{2}-6 ; \mathrm{H}-18$ with $\mathrm{H}_{2}-17$ and $\mathrm{H}_{2}-19$; $\mathrm{H}-$ 26 with $\mathrm{H}_{2}-25, \mathrm{H}-27$, and $\mathrm{H}_{2}-28$; and $\mathrm{H}_{3}-40$ with $\mathrm{H}_{2}-39$. The HMBC spectrum of 2 exhibited interactions of C-1 with $\mathrm{H}-2 ; \mathrm{C}-3$ with $\mathrm{H}-2, \mathrm{H}_{2}-6$, and $\mathrm{H}-4$; C-4 with $\mathrm{H}_{2}-5$ and $\mathrm{H}-3$; C-18 with $\mathrm{H}_{2}-17$ and $\mathrm{H}_{2}-19$; and $\mathrm{C}-26$ with $\mathrm{H}-27$ and $\mathrm{H}_{2}$-25. These evidences led to formulate the structure of $\mathbf{2}$ as $2 \alpha$-n-tetracont-(Z,Z)-3, 26-dien-18 $\alpha$-ol-1,5-olide. This is a new $\delta$-lactone isolated from a plant source.

Earlier $\delta$-lactonic constituents have been isolated from the root bark of Capparis deciduas [11], hulls of Oryza sativa [12], and seeds of Althea officinalis [13]. The compound 3 to 6 were the known phytoconstituents identified as glyceryl-1,2-dioctadec-9,12-dienoate-3-octadec-9,12-dienoate, glyceryl-1,2,3-trioctadecanoate, nnonadecanyl- $n$-docos-1-enoate, and $n$-octadec-9-enyl- $\beta$ D-glucopyranoside, respectively.

\section{Experimental}

\section{Plant material}

The fruits of C. sativum were collected from the herbal garden of Jamia Hamdard, New Delhi. The plant material was identified by Prof. MP Sharma, Taxonomist, Department of Botany, Faculty of Science, Jamia Hamdard, New Delhi. A voucher specimen (PRL/JH/07/27) of drug is 
preserved in the Phytochemistry Research Laboratory, Department of Pharmacognosy and Phytochemistry, Faculty of Pharmacy, Jamia Hamdard, New Delhi.

\section{Extraction}

C. sativum fruits $(1.6 \mathrm{~kg})$ were dried in air and then in an oven at $45^{\circ} \mathrm{C}$ temperature. The material was coarsely powdered. Exhaustive extraction of powdered drug was carried out in a Soxhlet apparatus using methanol as extracting solvent. The methanolic extract was concentrated under reduced pressure to yield a dark brown viscous mass, $187 \mathrm{~g}(11.68 \%)$.

\section{Isolation of phytoconstituents}

The methanolic extract (85 g) was dissolved in a minimum amount of methanol and adsorbed on silica gel $(60$ to 120 mesh) for preparation of slurry. The air-dried slurry was chromatographed over the silica gel column packed in petroleum ether $\left(60\right.$ to $\left.80^{\circ} \mathrm{C}\right)$. The column was eluted with petroleum ether $\left(60\right.$ to $\left.80^{\circ} \mathrm{C}\right)$, chloroform, and methanol in order of increasing polarity to isolate the following compounds:

\section{Coriander lactone (1)}

Elution of the column with petroleum ether furnished pale yellow crystals of 1, recrystallized from acetone, $76 \mathrm{mg}$ ( $0.089 \%$ yield); $R_{\mathrm{f}}$ value, 0.9 (petroleum ether); m.p, 110 to $111^{\circ} \mathrm{C}$; UV $\lambda_{\max }(\mathrm{MeOH}), 206 \mathrm{~nm}(\log € 4.9) ;$ IR $\gamma_{\max }$ (KBr), 2924, 2854, 1738, 1621, 1460, 1260, 1064, 806, $721 \mathrm{~cm}^{-1}$; ${ }^{1} \mathrm{H}$ NMR $\left(\mathrm{CDCl}_{3}\right), \delta 5.36(1 \mathrm{H}, \mathrm{dd}, J=6.6,7.2$, H-3), 5.32 (1 H, m, H-4), 4.14 (2 H, d, J=7.2 Hz), 2.78 ( $\left.1 \mathrm{H}, \mathrm{brm}, \mathrm{w}_{1 / 2}=6.1 \mathrm{~Hz}, \mathrm{H}-2 \beta\right), 2.30\left(2 \mathrm{H}, \mathrm{m}, \mathrm{H}_{2}-6\right), 2.05$ (4 H, m, $2 \times \mathrm{CH}_{2}$ ), $1.59\left(4 \mathrm{H}, \mathrm{m}, 2 \times \mathrm{CH}_{2}\right), 1.28$ (52 H, brs, $\left.26 \times \mathrm{CH}_{2}\right), 0.90(3 \mathrm{H}, \mathrm{t}, J=6.3, \mathrm{Me}-37) ;{ }^{13} \mathrm{C} \mathrm{NMR}\left(\mathrm{CDCl}_{3}\right)$, $\delta 173.56$ (C-1), 130.15 (C-3), 127.91 (C-4), 66.83 (C-5), $34.21 \quad(\mathrm{C}-2), \quad 31.90 \quad\left(\mathrm{CH}_{2}\right), \quad 29.65 \quad\left(21 \times \mathrm{CH}_{2}\right), \quad 29.33$ $\left(4 \times \mathrm{CH}_{2}\right), 27.19\left(\mathrm{CH}_{2}\right), 25.61\left(\mathrm{CH}_{2}\right), 24.90\left(\mathrm{CH}_{2}\right), 22.64$ $\left(\mathrm{CH}_{2}\right), 14.03$ (Me-37); +ve ion FAB MS m/z (rel. int.), 546 $[\mathrm{M}]^{+}\left(\mathrm{C}_{37} \mathrm{H}_{70} \mathrm{O}_{2}\right)$ (19.7), 449 (31.8), 97 (71.0).

\section{Hydroxy coriander lactone (2)}

Elution of the column with chloroform afforded pale yellow crystals of 2, recrystallized from acetone, $102 \mathrm{mg}(0.12 \%$ yield); $\mathrm{R}_{\mathrm{f}}$ value, 0.73 (chloroform); m.p., 95 to $96^{\circ} \mathrm{C}$; $\mathrm{UV}$ $\lambda_{\text {max }}(\mathrm{MeOH}), 205 \mathrm{~nm}(\log \in 5.1) ; \mathrm{IR} \gamma_{\max }(\mathrm{KBr}), 3463$, 2921, 2852, 1743, 1645, 1463, 1379, 1145, 1092, 1019, $722 \mathrm{~cm}^{-1} ;{ }^{1} \mathrm{H}$ NMR $\left(\mathrm{CDCl}_{3}\right), \delta 5.34(1 \mathrm{H}, \mathrm{dd}, J=6.8$, $7.5 \mathrm{~Hz}, \mathrm{H}-3), 5.30$ (1 H, m, H-4), 5.17 (1 H, brm, w $\mathrm{w}_{1 / 2}$ $=8.5 \mathrm{~Hz}, \mathrm{H}-26), 5.11$ ( $\left.1 \mathrm{H}, \mathrm{brm}, \mathrm{w}_{1 / 2}=9.5, \mathrm{H}-27\right), 4.32$ $(1 \mathrm{H}, \mathrm{d}, J=7.5 \mathrm{~Hz}, \mathrm{H}-5 \mathrm{a}), 4.28\left(1 \mathrm{H}, \mathrm{d}, J=7.5 \mathrm{~Hz}, \mathrm{H}_{2}-5 \mathrm{~b}\right)$, $4.16\left(1 \mathrm{H}, \mathrm{brm}, \mathrm{w}_{1 / 2}=5.7 \mathrm{~Hz}, \mathrm{H}-18 \beta\right), \delta 2.76\left(1 \mathrm{H}, \mathrm{m}, \mathrm{w}_{1 / 2}\right.$ $=6.8 \mathrm{~Hz}, \mathrm{H}-2 \beta), 2.30\left(2 \mathrm{H}, \mathrm{m}, \mathrm{H}_{2}-25\right), \delta 2.02\left(2 \mathrm{H}, \mathrm{m}, \mathrm{H}_{2}-\right.$ 28), $1.98\left(2 \mathrm{H}, \mathrm{m}, \mathrm{CH}_{2}\right), 1.68\left(2 \mathrm{H}, \mathrm{m}, \mathrm{CH}_{2}\right), 1.62(2 \mathrm{H}, \mathrm{m}$, $\left.\mathrm{CH}_{2}\right) .1 .60\left(2 \mathrm{H}, \mathrm{m}, \mathrm{CH}_{2}\right), 1.26\left(50 \mathrm{H}\right.$, brs, $\left.25 \times \mathrm{CH}_{2}\right), 0.87$
$(3 \mathrm{H}, \mathrm{t}, J=6.5 \mathrm{~Hz}, \mathrm{Me}-40) ;{ }^{13} \mathrm{C}$ NMR $\left(\mathrm{CDCl}_{3}\right), \delta 172.78(\mathrm{C}-$ 1), 130.34 (C-4), 129.78 (C-3), 128.78 (C-26), 127.96 (C-27), 68.83 (C-18), 65.95 (C-5), $42.03(\mathrm{C}-2), 33.76\left(\mathrm{CH}_{2}\right), 31.79$ $\left(\mathrm{CH}_{2}\right), \quad 31.68\left(\mathrm{CH}_{2}\right), 29.54\left(11 \times \mathrm{CH}_{2}\right), 29.21\left(5 \times \mathrm{CH}_{2}\right)$, $29.02\left(5 \times \mathrm{CH}_{2}\right), 27.67\left(\mathrm{CH}_{2}\right), 27.11\left(\mathrm{CH}_{2}\right), 26.66\left(\mathrm{CH}_{2}\right)$, $25.50\left(\mathrm{CH}_{2}\right), 24.73\left(\mathrm{CH}_{2}\right), 24.34\left(\mathrm{CH}_{2}\right), 22.55\left(\mathrm{CH}_{2}\right), 13.94$ (Me-40); +ve ion FAB MS $m / z$ (rel. int.), $603[\mathrm{M}+\mathrm{H}]^{+}$ $\left(\mathrm{C}_{40} \mathrm{H}_{75} \mathrm{O}_{3}\right.$ ) (100), 559 (3.7), 503 (6.1), 461 (3.6), 405 (3.8), 393 (18.0), 337 (18.2), 295 (14.1), 265 (39.8), 97 (37.9).

\section{Glyceryl-1,2-dioctadec-9,12-dienoate-3-octadec-9-enoate (3)} Elution of column with chloroform-methanol (19:1) afforded light green resinous mass of 3, crystallized from acetone, $82 \mathrm{mg}\left(0.096 \%\right.$ yield); $\mathrm{R}_{\mathrm{f}}$ value, 0.6 (chloroformmethanol, 19: 1); m.p., 65 to $66^{\circ} \mathrm{C}$; UV $\lambda_{\max }(\mathrm{MeOH})$, $206 \mathrm{~nm}\left(\log €\right.$ 4.6); IR $\gamma_{\max }(\mathrm{KBr}), 2925,2855,1743$, 17251640, 1460, 1375, 1167, $723 \mathrm{~cm}^{-1} ;{ }^{1} \mathrm{H}$ NMR $\left(\mathrm{CDCl}_{3}\right), \delta$ 5.34 (4 H, m, H-9', H-13', H-9' ', H-13' '), 5.32 (4 H, m, H10 ', H-12', H-10' ', H-12' '), 5.27 ( $2 \mathrm{H}, \mathrm{m}, \mathrm{H}-9^{\prime \prime}$ ' ', H-10' ' '), $4.33(1 \mathrm{H}, \mathrm{d}, J=1.7 \mathrm{~Hz}, \mathrm{H}-2)$, $4.14\left(2 \mathrm{H}, \mathrm{m}, \mathrm{H}_{2}-1\right), 4.11(2 \mathrm{H}$, m, $\left.\mathrm{H}_{2}-3\right), 2.31\left(1 \mathrm{H}, \mathrm{d}, J=3.0 \mathrm{~Hz}, \mathrm{H}_{2}-2^{\prime} \mathrm{a}\right), \delta 2.30(1 \mathrm{H}, \mathrm{d}$, $\left.J=3.0 \mathrm{~Hz}, \mathrm{H}_{2}-2^{\prime} \mathrm{b}\right), 2.20$ (1 H, d, $\left.J=6.3 \mathrm{~Hz}, \mathrm{H}_{2}-2^{\prime \prime}{ }^{\prime} \mathrm{a}\right), \delta 2.18$ $\left(1 \mathrm{H}, \mathrm{d}, J=6.3 \mathrm{~Hz}, \mathrm{H}_{2}-2^{\prime \prime}\right.$ 'b), $2.16\left(1 \mathrm{H}, \mathrm{d}, J=7.5 \mathrm{~Hz}, \mathrm{H}_{2}-2^{\prime \prime}\right.$ ' a), 2.14 (1 H, d, J=7.5 Hz, H $2^{2}{ }^{\prime \prime \prime}$ b), 1.85 ( $\left.2 \mathrm{H}, \mathrm{m}, \mathrm{H}_{2}-11^{\prime}\right)$. 1.81 ( $\left.2 \mathrm{H}, \mathrm{m}, \mathrm{H}_{2}-11^{\prime \prime}\right), 1.79$ (4 $\left.\mathrm{H}, \mathrm{m}, \mathrm{H}_{2}-8^{\prime}, \mathrm{H}-8^{\prime \prime}\right), 1.77$ (4 H, m, H2-14', $\mathrm{H}_{2}-14^{\prime \prime}$ ), 1.61 (4 H, m, $\mathrm{H}_{2}-8^{\prime \prime \prime}, \mathrm{H}_{2}-11^{\prime \prime \prime}$ '), $1.26\left(48 \mathrm{H}\right.$, brs, $\left.24 \times \mathrm{CH}_{2}\right), 1.16\left(4 \mathrm{H}, \mathrm{m}, 2 \times \mathrm{CH}_{2}\right), 0.87(9 \mathrm{H}$, m, $\left.\mathrm{H}_{3}-18^{\prime}, \mathrm{H}_{3}-18^{\prime \prime}, \mathrm{H}_{3}-18^{\prime \prime \prime}\right)$; ${ }^{13} \mathrm{C} \mathrm{NMR}\left(\mathrm{CDCl}_{3}\right) \delta 173.37$ $\left(\mathrm{C}-1^{\prime}\right), 172.80$ (C-1' $), 172.49$ (C-1'"'), 130.22 (C-10', C$\left.10^{\prime \prime}\right), 129.83$ (C-12'), 129.66 (C-12' $), 128.68$ (C-9'"', C$\left.10^{\prime \prime \prime}\right), 128.68$ (C-9', C-9' ') 127.82 (C-13'), 127.68 (C-13' '), 68.77 (C-2), 64.72 (C-1), 61.87 (C-3), 56.93 (C-2'), 56.62 (C-

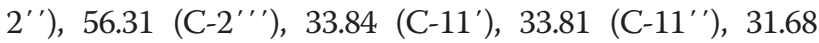
$\left(2 \times \mathrm{CH}_{2}\right), 31.28\left(\mathrm{CH}_{2}\right), 29.42\left(10 \times \mathrm{CH}_{2}\right), 29.09\left(5 \times \mathrm{CH}_{2}\right)$, $28.91\left(5 \times \mathrm{CH}_{2}\right), 27.54\left(\mathrm{CH}_{2}\right), 26.96\left(\mathrm{CH}_{2}\right), 26.55\left(\mathrm{CH}_{2}\right)$, $25.94\left(\mathrm{CH}_{2}\right), 25.38\left(\mathrm{CH}_{2}\right), 28.58\left(\mathrm{CH}_{2}\right), 24.23\left(\mathrm{CH}_{2}\right), 22.42$ (3× $\left.\mathrm{CH}_{2}\right), 13.80\left(\mathrm{Me}-18^{\prime}, \mathrm{Me}-18^{\prime \prime}, \mathrm{Me}-18^{\prime \prime \prime}\right)$; +ve ion FAB MS $m / z$ (rel. int.), $880[\mathrm{M}]^{+}\left(\mathrm{C}_{57} \mathrm{H}_{100} \mathrm{O}_{6}\right)$ (1.5), 617 (29.8), 602 (37.0), 339 (71.8), 337 (31.1), 281 (21.5), 279 (31.6), 265 (24.8), 137 (26.2), 111 (39.9).

\section{Glyceryl-1,2,3-trioctadecanoate (4)}

Elution of column with chloroform-methanol (97: 3) furnished a colorless resinous mass of $\mathbf{4}$, crystallized from from acetone, $132 \mathrm{mg}\left(0.155 \%\right.$ yielded); $R_{\mathrm{f}}$ value, 0.23 (chloroform-methanol, 22: 3); m.p. 86 to $88^{\circ} \mathrm{C}$; UV $\lambda_{\max }$ $(\mathrm{MeOH}), 207 \mathrm{~nm}(\log \in 4.6)$; IR $\gamma_{\max }(\mathrm{KBr}), 2924,2854$, $1725,1721,1459,1373,1265,1170,723 \mathrm{~cm}^{-1} ;{ }^{1} \mathrm{H}$ NMR (DMSO-d6), $\delta 3.63$ (1 H, m, H-2), $3.50\left(2 \mathrm{H}, \mathrm{m}, \mathrm{H}_{2}-1\right)$, 3.23 ( $2 \mathrm{H}, \mathrm{m}, \mathrm{H}_{2}-3$ ), 2.49 ( $2 \mathrm{H}$, brs, $\mathrm{H}_{2}-2^{\prime \prime}$ ), 2.25 ( $2 \mathrm{H}$, brs, $\left.\mathrm{H}_{2}-2^{\prime}\right), 2.18$ (2 H, brs, $\left.\mathrm{H}_{2}-2^{\prime \prime \prime}\right), 2.05\left(2 \mathrm{H}, \mathrm{m}, \mathrm{CH}_{2}\right), 1.98$ $\left(4 \mathrm{H}, \mathrm{m}, 2 \times \mathrm{CH}_{2}\right), 1.70\left(2 \mathrm{H}, \mathrm{m}, \mathrm{CH}_{2}\right), 1.61(4 \mathrm{H}, \mathrm{m}$, $\left.2 \times \mathrm{CH}_{2}\right), 1.49\left(6 \mathrm{H}, \mathrm{m}, 3 \times \mathrm{CH}_{2}\right), 1.31\left(8 \mathrm{H}\right.$, brs, $\left.4 \times \mathrm{CH}_{2}\right)$, $1.22\left(20 \mathrm{H}\right.$, brs, $\left.10 \times \mathrm{CH}_{2}\right), 1.20\left(18 \mathrm{H}\right.$, brs, $\left.9 \times \mathrm{CH}_{2}\right), 1.18$ 
$\left(18 \mathrm{H}\right.$, brs, $\left.9 \times \mathrm{CH}_{2}\right), 1.16\left(8 \mathrm{H}\right.$, brs, $\left.4 \times \mathrm{CH}_{2}\right), 0.84(9 \mathrm{H}$, brs, $\left.3 \times \mathrm{CH}_{3}\right) ;{ }^{13} \mathrm{C}$ NMR (DMSO-d6), $\delta 174.51\left(\mathrm{C}-1^{\prime \prime}\right)$, 173.80 (C-1'), $172.72\left(\mathrm{C}-1^{\prime \prime \prime}\right), 71.40$ (C-2), 69.89 (C-1), 64.81 (C-3), $56.67\left(\mathrm{C}-1^{\prime \prime}\right), 56.67\left(\mathrm{C}-1^{\prime}\right), 51.35\left(\mathrm{C}-1^{\prime \prime \prime}\right)$, $33.73\left(\mathrm{CH}_{2}\right), \quad 32.62\left(\mathrm{CH}_{2}\right), \quad 29.19\left(20 \times \mathrm{CH}_{2}\right), \quad 28.87$ $\left(16 \times \mathrm{CH}_{2}\right), 27.73\left(\mathrm{CH}_{2}\right), 26.70\left(\mathrm{CH}_{2}\right), 25.21\left(\mathrm{CH}_{2}\right), 24.55$ $\left(\mathrm{CH}_{2}\right), 22.20\left(3 \times \mathrm{CH}_{2}\right)$; +ve ion FAB MS $m / z$ (rel. int.), $890[\mathrm{M}]^{+}\left(\mathrm{C}_{57} \mathrm{H}_{110} \mathrm{O}_{6}\right)$ (1.5), 283 (15.1), 267 (18.3).

\section{n-nonadecanyl-n-docos-11-enoate (5)}

Elution of column with chloroform-methanol (91:9) afforded light brown mass resinous mass of $\mathbf{5}$, crystallized from acetone, $112 \mathrm{mg}$ ( $0.131 \%$ yield); $R_{\mathrm{f}}$ value, 0.44 (chloroform-methanol, 22: 3); m.p., 71 to $73^{\circ} \mathrm{C}$; UV $\lambda_{\max }$ $(\mathrm{MeOH}), 207 \mathrm{~nm}(\log \in 4.3)$; IR $\gamma_{\max }(\mathrm{KBr}), 2924,2854$, $1721,1459,1373,1265,1170,723 \mathrm{~cm}^{-1} ;{ }^{1} \mathrm{H}$ NMR $\left(\mathrm{CDCl}_{3}\right), \delta$ 5.34 (2 H, m, H-11. H-12), $3.79(1 \mathrm{H}, \mathrm{d}$, $\left.J=9.9 \mathrm{~Hz}, \mathrm{H}_{2}-1^{\prime} \mathrm{a}\right), 3.75\left(1 \mathrm{H}, \mathrm{d}, J=9.9 \mathrm{~Hz}, \mathrm{H}_{2}-1^{\prime} \mathrm{b}\right), 2.62$ (2 H, brs, $\left.\mathrm{H}_{2}-2\right)$, 2.30 ( $\left.2 \mathrm{H}, \mathrm{m}, \mathrm{H}_{2}-10\right), 2.03\left(2 \mathrm{H}, \mathrm{m}, \mathrm{H}_{2}\right.$ 13), $1.60\left(4 \mathrm{H}, \mathrm{m}, 2 \times \mathrm{CH}_{2}\right), 1.25\left(60 \mathrm{H}\right.$, brs, $\left.30 \times \mathrm{CH}_{2}\right)$, $0.87(3 \mathrm{H}, \mathrm{t}, J=6.3 \mathrm{~Hz}, \mathrm{Me}-22), 0.84(3 \mathrm{H}, \mathrm{t}, J=6.1 \mathrm{~Hz}$, Me-19'); ${ }^{13} \mathrm{C}$ NMR $\left(\mathrm{CDCl}_{3}\right), \delta 172.51$ (C-1), 130.01 (C11), 116.02 (C-12), 65.16 (C-1'), 51.74 (C-2), 32.88 $\left(\mathrm{CH}_{2}\right), 31.76\left(\mathrm{CH}_{2}\right), 29.53\left(20 \times \mathrm{CH}_{2}\right), 29.20\left(9 \times \mathrm{CH}_{2}\right)$, $27.07\left(\mathrm{CH}_{2}\right), 24.53\left(\mathrm{CH}_{2}\right), 22.52\left(\mathrm{CH}_{2}\right), 13.94(\mathrm{Me}-22$, Me- 19'); +ve ion FAB MS $m / z$ (rel. int.), 604[M $]^{+}$ $\left(\mathrm{C}_{41} \mathrm{H}_{80} \mathrm{O}_{2}\right)$ (23.1), 337 (31.0), 267 (22.5).

\section{Oleiyl glycoside (6)}

Elution of column with chloroform-methanol (22: 3) afforded colorless mass of $\mathbf{6}$, crystallized from acetone, $145 \mathrm{mg}\left(0.170 \%\right.$ yield); $R_{\mathrm{f}}$ value, 0.2 (chloroform-methanol, 17: 3); m.p., 60 to $62^{\circ} \mathrm{C}$; UV $\lambda_{\max }(\mathrm{MeOH}), 209 \mathrm{~nm}$ $(\log € 5.3) ; \mathrm{IR} \gamma_{\max }(\mathrm{KBr}), 3410,3360,2925,2855,1733$, 1640, 1457, 1261, 1091, $801 \mathrm{~cm}^{-1} ;{ }^{1} \mathrm{H}$ NMR $\left(\mathrm{CDCl}_{3}\right), \delta$ 5.31 (2 H, m, H-9, H-10), 5.25 (1 H, brs, H-1'), 4.41 (1 H, m, H-5'), 4.19 (1 H, m, H-2'), 3.61 (1 H, m, H-3'), 3.58 (1 H, m, H-4' ), 3.27 (2 H, brs, $\mathrm{H}_{2}-6^{\prime}$ ), 2.67 (2 H, m, $\left.\mathrm{H}_{2}-2\right), 2.18\left(2 \mathrm{H}, \mathrm{m}, \mathrm{H}_{2}-8\right), 1.93$ (2 $\left.\mathrm{H}, \mathrm{m}, \mathrm{H}_{2}-11\right), 1.48$ (4 $\mathrm{H}$, brs, $\left.2 \times \mathrm{CH}_{2}\right), 1.16\left(18 \mathrm{H}\right.$, brs, $\left.9 \times \mathrm{CH}_{2}\right), 0.77(3 \mathrm{H}$, $\mathrm{t}, J=6.1 \mathrm{~Hz}, \mathrm{Me}-18) .{ }^{13} \mathrm{C} \mathrm{NMR}\left(\mathrm{CDCl}_{3}\right), \delta 173.67(\mathrm{C}-1)$, 129.85 (C-9), 127.71 (C-10), 103.15 (C-1'), 80.23 (C-5'), 69.83 (C- 2'), 68.06 (C-3'), 67.08 (C-4'), 61.05 (C-6'), $55.67(\mathrm{C}-2), 52.27\left(\mathrm{CH}_{2}\right), 38.55\left(\mathrm{CH}_{2}\right), 33.74\left(\mathrm{CH}_{2}\right)$, $31.57\left(\mathrm{CH}_{2}\right), \quad 29.34\left(3 \times \mathrm{CH}_{2}\right), 29.02\left(2 \times \mathrm{CH}_{2}\right), \quad 26.89$ $\left(\mathrm{CH}_{2}\right), 25.31\left(\mathrm{CH}_{2}\right), 24.55\left(\mathrm{CH}_{2}\right), 22.34\left(\mathrm{CH}_{2}\right), 13.78$ (Me-18); +ve ion FAB MS $m / z$ (rel. int.), $445[\mathrm{M}+\mathrm{H}]^{+}$ $\left(\mathrm{C}_{24} \mathrm{H}_{45} \mathrm{O}_{7}\right)$ (35.6), 265 (28.3), 180 (26.7).

\section{Conclusions}

Phytochemical investigation of fruits of C. sativum led to isolate new aliphatic $\delta$-lactones which may be used as chromatographic markers for quality control of the drugs.

\section{Additional file}

Additional file 1: Showing spectrum of ${ }^{1} \mathrm{H}$ NMR and ${ }^{13} \mathrm{C}$ NMR of coriander lactone and hydroxy coriander lactone and mass spectrum of hydroxy coriander lactone. The file contains ${ }^{1} \mathrm{H} N M R,{ }^{13} \mathrm{C}$ NMR, and mass spectrum of coriander lactone and hydroxyl coriander lactone.

\section{Competing interests}

The authors declare that they have no competing interests.

\section{Acknowledgments}

The authors are thankful to the Head, Sophisticated Instrumentation Analytical Facility, Central Drug Research Institute, Lucknow, for recording the mass spectra of the compounds.

Received: 15 November 2011 Accepted: 16 July 2012 Published: 16 July 2012

\section{References}

1. Innocent BX, Fathima MSA, Dhanalakshmi (2011) Studies on the immouostimulant activity of Coriandrum sativum and resistance to Aeromonas hydrophila in Catla catla. J Appl Pharmaceut Sci 1(7):132-135

2. Anonymous (2001) Wealth of India, aw aterial. National Institute of Science Communication, CSIR, New Delhi, pp 203-206, 2

3. Pathak NL, Kasture SB, Bhatt NM, Rathod JD (2011) Phytopharmacological properties of Coriander sativum as a potential medicinal tree: an overview. J Appl Pharmaceut Sci 1(4):20-25

4. Coskuner Y, Karababa E (2007) Physical properties of coriander seed (Coriandrum sativum L.). J Food Eng 80(2):408-416

5. Eikani MH, Golmohammad F, Rowshanzamir S (2007) Supercritical water extraction of essential oils from coriander seeds (Corinadrum sativum L.). J Food Eng 80(2):735-740

6. Helle W, Anne BS, Karl EM (2004) Antioxidant activity in extracts from coriander. Food Chem 88(2):293-297

7. Taniguchi M, Yanai M, Xiao YQ, Kido T, Baba K (1996) Three isocoumarins from Coriandrum sativum. Phytochemistry 42(3):843-846

8. Gray AM, Flatt PR (1999) Insulin-releasing and insulin-like activity of the traditional anti-diabetic plant Coriandrum sativum (coriander). Br J Nutr 81 (3):203-209

9. Chithra V, Leelamma S (1997) Hypolipidemic effect of coriander seeds (Coriandrum sativum): mechanism of action. Plant Foods Hum Nutr 51 (2):167-172

10. Lal AA, Kumar T, Murthy PB, Pillai KS (2004) Hypolipidemic effect of Coriandrum sativum L. in triton-induced hyperlipidemic rats. Indian J Exp Biol 42(9):909-912

11. Gupta J, Ali M (1997) Oxygenated heterocyclic constituents from Capparis deciduas root barks. Indian J Heterocyclic Chem 6(4):295-302

12. Chung IM, Ali M, Chun S-C, Lee O-K, Ahmad A (2007) Sativalanosteronyl glycoside and oryzatriacontolide constituents from the Hulls of Oryza sativa. Asian J Chem 19(2):1535-1543

13. Reni S, Khan SA, Ali M (2010) Phytochemical investigation of the seeds of Althea officinalis L. Nat Prod Res 24(14):1358-1364

doi:10.1186/2191-2858-2-28

Cite this article as: Naquvi et al.: Two new aliphatic lactones from the fruits of Coriandrum sativum L.. Organic and Medicinal Chemistry Letters 2012 2:28. 